\title{
Organizational Contact Postal Address
}

National Cancer Institute

\section{Source}

National Cancer Institute. Organizational Contact Postal Address. NCI Thesaurus. Code C93880.

A contact point used to send physical forms of communication to the organizational contact. 